\title{
Greater asymmetric wall shear stress in Sievers' type 1/LR compared with 0/LAT bicuspid aortic valves after valve-sparing aortic root replacement
}

\author{
Elizabeth H. Stephens, MD, PhD, ${ }^{\mathrm{a}}$ Thomas A. Hope, MD, ${ }^{\mathrm{b}}$ Fabian A. Kari, MD, \\ John-Peder Escobar Kvitting, MD, PhD, ${ }^{d}$ David H. Liang, MD, PhD, ${ }^{e}$ Robert J. Herfkens, MD, ${ }^{b}$ and \\ D. Craig Miller, $\mathrm{MD}^{\mathrm{f}}$
}

\begin{abstract}
Objective: To evaluate the role of commissure orientation on downstream blood flow patterns and ascending aortic wall shear stress (WSS) in patients with bicuspid aortic valves (BAV) after valve-sparing aortic root replacement (V-SARR).
\end{abstract}

Methods: Nineteen BAV patients after V-SARR (9 Sievers' type 1/LR [type 1 valve with fusion of the left and right cusps] and 10 Sievers' type 0/LAT ["naturally perfect"; type 0 valve without the presence of a raphe, and with the 2 commissures oriented right-anterior-to-left-posterior]) were imaged using time-resolved 3-D phase contrast magnetic resonance imaging. A control group of 5 unoperated tricuspid aortic valve patients were used for comparison purposes. Wall shear stress and eccentricity of flow normalized to aortic diameter were measured in planes placed perpendicular to the axis of the ascending aorta at the level of the sinotubular junction (proximal ascending), main pulmonary artery (mid-ascending), and origin of the brachiocephalic (distal ascending).

Results: The ratio of WSS along the outer curvature to that along the inner curvature was greater in Sievers' type 1/LR patients compared with Sievers' type 0/LAT patients in the proximal $(3.8 \pm 1.6 \mathrm{vs} 2.1 \pm 0.9, P=.009)$ and mid- ascending aorta ( $4.5 \pm 2.4$ vs $2.4 \pm 1.3, P=.027)$. Relative to control normal tricuspid patients, Sievers' type 1/LR BAV patients had a higher WSS ratio in the mid-ascending aorta $(4.5 \pm 2.4$ vs $1.2 \pm 1.2, P=.007)$. Conversely, WSS in Sievers' type 0/LAT patients was not different than in normal tricuspid patients.

Conclusions: After V-SARR, BAV cusp morphology has a major impact on the pattern of blood flow and WSS in the ascending aorta. ( $\mathrm{J}$ Thorac Cardiovasc Surg 2015;150:59-68)

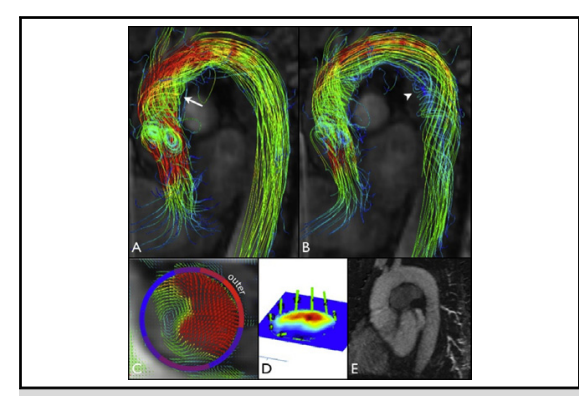

Altered flow in Sievers' type 1/LRBAV: (A, B) streamlines; (C) vector fields; (D) WSS; and (E) postoperative MRA.

\section{Central Message}

Sievers' type 1/LR BAV patients, after V-SARR, had greater asymmetric ascending aortic WSS and flow eccentricity than did Sievers" type 0/LAT ("naturally perfect") BAV patients, and greater WSS compared with control patients with a tricuspid aortic valve.

\section{Perspective}

Altered flow combined with abnormal aortic composition in BAV patients lead to aortic pathologies such as dilatation and dissection. Differences between BAV cusp morphology in terms of valvular disease progression, flow patterns, and aortopathy have been demonstrated, but studies examining flow after V-SARR, in which the remaining native aorta remains at risk for complications, are lacking. We examined flow in Sievers' type $1 / \mathrm{LR}$ and 0/LAT patients after V-SARR and found that type 1/LR patients had greater asymmetric ascending aortic wall shear stress and flow eccentricity than did 0/LAT BAV patients and control tricuspid patients.

See Editorial Commentary page 68.

\footnotetext{
From the a Division of Cardiac, Thoracic, and Vascular Surgery, New York Presbyterian Hospital, Columbia University Medical Center, New York, NY; ${ }^{\mathrm{b}}$ Department of Radiology, Stanford University School of Medicine, Stanford, Calif; ${ }^{\mathrm{c}}$ Department of Cardiovascular Surgery, Heart Center, University of Freiburg, Freiburg, Germany; ${ }^{\mathrm{d} D e p a r t m e n t}$ of Cardiothoracic Surgery, Linköping University Hospital, Linköping, Sweden; ${ }^{\mathrm{e} D i v i s i o n}$ of Cardiovascular Medicine, and ${ }^{\mathrm{f}}$ Department of Cardiovascular and Thoracic Surgery, Stanford University School of Medicine, Stanford, Calif.

E.H. Stephens was supported by a Carl and Leah McConnell Cardiovascular Surgical Research Fellowship.
}

Read at the 39th Annual Meeting of The Western Thoracic Surgical Association, Coeur d'Alene, Idaho, June 26-29, 2013.

Received for publication Jan 2, 2015; revisions received March 31, 2015; accepted for publication April 7, 2015; available ahead of print May 6, 2015.

Address for reprints: D. Craig Miller, MD, Department of Cardiovascular and Thoracic Surgery, Falk Cardiovascular Research Center, Stanford University School of Medicine, Stanford, CA 94305-5247 (E-mail: dcm@stanford.edu). $0022-5223 / \$ 36.00$

Copyright (C) 2015 by The American Association for Thoracic Surgery http://dx.doi.org/10.1016/j.jtcvs.2015.04.020 


$\begin{array}{ll}\text { Abbreviations and Acronyms } \\ \text { BAV } & \text { bicuspid aortic valve } \\ \text { CT } & \text { computed tomography } \\ \text { Type 0/LAT = } & \text { type } 0 \text { valve without the presence of } \\ & \text { a raphe, and with the } 2 \text { commissures } \\ & \text { oriented right-anterior-to-left- } \\ & \text { posterior } \\ = & \text { type } 1 \text { valve with fusion of the left } \\ & \text { and right cusps with } 1 \text { raphe } \\ = & \text { type } 1 \text { valve with fusion of the right } \\ & \text { and noncoronary cusps with } 1 \text { raphe } \\ = & \text { valve-sparing aortic root } \\ & \text { replacement } \\ = & \text { wall shear stress } \\ \text { Type 1/RN } & \text { time-resolved 3-dimensional phase } \\ & \text { contrast magnetic resonance } \\ \text { V-SARR } & \text { imaging } \\ \text { WSS } & \end{array}$

Bicuspid aortic valve (BAV) disease is the most common congenital heart defect and is associated with aortic dilatation and increased risk of aortic rupture or dissection. The aortic root and ascending aorta in patients with a BAV demonstrate distinct patterns of dilation. ${ }^{1}$ Altered hemodynamics is one possible explanation for aortic dilation in BAV disease; however, aortic dilation in patients with normal BAV function ${ }^{2}$ dispelled the notion that abnormal flow was solely responsible. Investigations using time-resolved 3-dimensional phase contrast magnetic resonance imaging (4D flow MRI) demonstrated altered fluid dynamics even in BAV patients without valve dysfunction. $^{3-5}$ Different BAV cusp morphologies additionally have distinctly different flow patterns, ${ }^{3-6}$ as well as distinct patterns of aortopathy and clinical progression. ${ }^{7-9}$ These eccentric flow profiles in BAV patients are associated with elevated aortic wall shear stress (WSS), ${ }^{5,6,10}$ and aortic dilation is related to degree of ascending aortic flow eccentricity. ${ }^{11}$

Over the past decade, interest has increased in valve-sparing aortic root replacement (V-SARR) for BAV patients; although midterm results are excellent, the long-term outcomes are unknown. ${ }^{12}$ Complications seen after V-SARR in tricuspid aortic valve patients, such as valvular degeneration and dissection of the distal aorta, are likely to also occur in BAV patients, as well as progressive sclerosis of the BAV. Altered flow patterns associated with a BAV will not affect the graft, but potentially may affect the risk of complications in the downstream native aorta.

The objective of this study was to examine the influence of BAV commissural orientation on ascending aortic blood flow and WSS after V-SARR, by comparing the flow patterns and WSS of patients with Sievers' type 0/LAT (type
0 valve without the presence of a raphe, and with the 2 commissures oriented right-anterior-to-left-posterior) and type 1/LR (type 1 valve with fusion of the left and right cusps) BAVs (Figure 1). We hypothesized that Sievers' type 1/ LR patients have increased WSS, compared with 0/LAT BAV patients, after V-SARR, owing to the orientation of the aortic systolic flow toward the outer curvature in type 1/LR patients.

\section{METHODS \\ Study Group}

This investigation was approved by the institutional review board, and informed consent was obtained. All patients had undergone a Tirone David V V-SARR with Stanford modification, as described previously. ${ }^{13}$ Classification of BAV using the Sievers' system was based on intraoperative findings. ${ }^{14}$ Nineteen patients were imaged using 4D flow MRI. Nine patients had a Sievers' type 1 valve with fusion of the left and right cusps (1/LR); 10 patients had a Sievers' type 0 valve without the presence of a raphe, and with the 2 commissures oriented right-anterior-to-left-posterior (0/LAT; see Figure 1 and Table 1). Previously collected 4D flow MRI data ${ }^{15}$ from 5 unoperated healthy subjects with tricuspid aortic valves ( 3 men and 2 women; mean age: $33.2 \pm 13.6$ years), with normal valve function, no cardiac disease, and normal aortic diameters, were used as a control.

Postoperative CT scans were used to calculate cusp angle. Axial CT scan slices were manually screened for the slice that most clearly demonstrated the aortic cusps, commissures, and free margins. On that image, cusp angles were measured manually using commercially available software (UTHSCSA ImageTool; University of Texas Health Science Center-San Antonio, San Antonio, Tex), as described previously. ${ }^{16}$

\section{D Flow Imaging Technique}

All imaging studies were performed on a 3.0-T system (MR750, GE Healthcare, Milwaukee, Wis) using a 32-channel cardiac coil. The 4D flow acquisition was acquired in the coronal plane using the following imaging parameters: bandwidth $= \pm 62.5 \mathrm{kHz}$; flip angle $=20$; echo time/repetition time $=1.5 / 4.5$; number of averages $=1$; field of view $=400 \times 400 \mathrm{~mm}^{2}$; matrix size $=256 \times 256 \times 120$; and spatial resolution $=1.6 \times 1.6 \times 1.6 \mathrm{~mm}$. The temporal resolution of the data equaled $16 \times$ repetition time. Three-directional velocity encoding was utilized, with the velocity encoding ranging from 150 to $300 \mathrm{~cm} / \mathrm{second}$, depending on the peak velocity in the ascending aorta. Aliasing, if present, was manually corrected for using a Matlab script (Matlab, MathWorks, Natick, Mass). Gadofosveset trisodium (Ablavar, Lantheus Medical Imaging, Billerica, Mass) was administered at a dose of $0.03 \mathrm{mmol} / \mathrm{kg}$ before the acquisition. Cardiac gating was obtained using vector electrocardiography with an average heart rate of $61 \pm 7.9$ beats per minute. Respiratory compensation with $\mathrm{k}$-space phase reordering (EXORCIST, GE Healthcare, Milwaukee, Wis) was utilized. To decrease the scan time, imaging was accelerated using a combination of parallel imaging and compressed sensing, ${ }^{17}$ resulting in an average scan time of $13.7 \pm 1.9$ minutes. Before analysis, images were corrected for Maxwell phase effects, encoding errors related to gradient field distortions, and eddy current-related phase offsets.

\section{Qualitative Flow Evaluation}

Flow throughout the thoracic aorta was qualitatively analyzed using vector fields, streamlines, and particle traces, using EnSight (CEI, Apex, $\mathrm{NC}$ ) in the ascending aorta, transverse aortic arch, and descending aorta, based on criteria adapted from previously published criteria. ${ }^{18}$ To determine the degree of rotation, particle traces released at the 

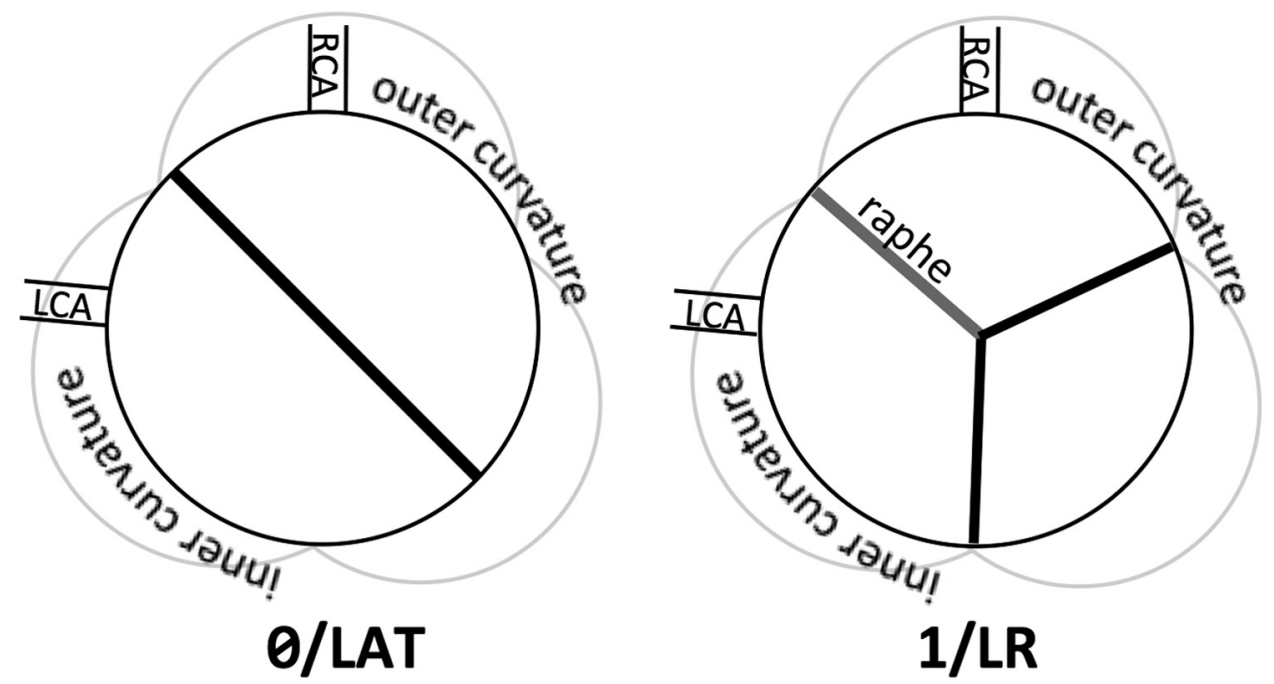

FIGURE 1. Diagrammatic representation of cusp orientation in Sievers' type 1/LR and 0/LAT bicuspid aortic valve patients. Sievers' type 0/LAT patients (left) have symmetric cusps, with the commissures spanning the LCA and RCA. Sievers' type 1/LR patients have commissural fusion of the left and right cusps with a raphe. Note the relationship of the valve orifice in each group to the curvature of the ascending aorta. $L C A$, Left coronary artery; $R C A$, right coronary artery; $0 / L A T$, type 0 valve without the presence of a raphe, and with the 2 commissures oriented right-anterior-to-left-posterior; $1 / L R$, type 1 valve with fusion of the left and right cusps.

beginning of systole were visualized qualitatively to determine the degree of helical flow. Additionally, the percentage of the vessel lumen containing helical flow was quantified based on axial vector fields at multiple planes through the ascending aorta.

Finally, helical flow was evaluated for directionality and categorized as either a right-handed (counterclockwise) or left-handed (clockwise) helix, as viewed distal to the flow, ie, from the distal ascending aorta looking toward the aortic valve, using vector fields on axial planes through the ascending aorta. Helical flow describes flow that rotates around an axis with net forward flow. Streamlines during peak systole were visualized to determine the presence of vortical flow, which describes rotational flow that does not have a net forward flow. Vortical flow in the sinuses of Valsalva was not included in this analysis. Helical and vortical flow was graded as none $(0),<360^{\circ}$ of rotation (1), or $>360^{\circ}$ of rotation (2).

\section{Quantitative Flow and Shear Stress Analysis}

Using EnSight, planes were placed in the proximal ascending aorta at the level of the sinotubular junction (proximal ascending), the mid-ascending aorta at the level of the main pulmonary artery, and in the distal ascending aorta just proximal to the origin of the brachiocephalic artery. In each plane, the ascending aorta was manually segmented, and vessel eccentricity and WSS were evaluated. For eccentricity, displacement of the flow jet from the vessel center, normalized to vessel diameter, was used. For WSS, the vessel wall was divided into 12 segments; the ratio of the average shear of the 3 segments along the outer curvature and the 3 segments along the inner curvature was calculated as previously described (flow tool, University of Freiburg, Freiburg, Germany). ${ }^{19}$

\section{Statistical Analysis}

Scalar data are presented as mean \pm 1 standard deviation. Nominal variables are presented as number and frequency. Groups were compared using the Fisher exact test for nominal variables and the Wilcoxon test for scalar variables. For the comparison of WSS ratios and eccentricity at each level in the ascending aorta, the Wilcoxon rank-sum test was used. To compare BAV to tricuspid patients, the Kruskal-Wallis test was used, and if significant, post hoc testing was performed using the Mann-Whitney $U$ test with Bonferroni correction. All statistical testing was performed using commercially available software (SPSS, Version 17; SPSS, Inc, Chicago, Ill). Given the potential role of aortic stenosis affecting flow parameters and WSS, additional analysis was performed, excluding the patients with moderate aortic stenosis (defined as mean pressure gradient $>20 \mathrm{~mm} \mathrm{Hg}$ and/or maximum velocity $>3.0 \mathrm{~m} / \mathrm{second}$ on echocardiogram).

\section{RESULTS \\ Patient Demographics}

The mean age of BAV patients at the time of operation was $45 \pm 9.4$ years (Table 1); age at time of MRI was $48.7 \pm 9.9$ years, and imaging was performed an average of $51 \pm 32$ months postoperatively. Sixteen of the patients $(84 \%)$ were men. Although no statistically significant differences were found between Sievers' type 1/LR and 0/LAT patients in preoperative or intraoperative characteristics, Sievers' type 1/LR patients had a significantly higher mean pressure gradient and maximum velocity (Table 1); however, when additional analysis was performed, excluding the 3 patients with mild-moderate aortic stenosis, the only statistically significant difference between groups was the postrepair annular diameter, which was significantly larger in the Sievers' type 1/LR patients $(26.2 \pm 3.6$ vs $21.8 \pm 1.2 \mathrm{~mm}, P=.012)$.

\section{Qualitative Flow Analysis}

In the ascending aorta, all 10 patients with Sievers' type 0/LAT, and 7 of the 9 with Sievers' type 1/LR BAVs demonstrated helical flow that involved $52 \% \pm 29 \%$ of the cross-sectional area of the ascending aorta during peak systole (Table 2). Three of the 10 Sievers' type 0/LAT patients had flattened aortic jets, which was 
TABLE 1. Patient characteristics

\begin{tabular}{|c|c|c|c|}
\hline Characteristic & 0/LAT $(\mathbf{n}=\mathbf{1 0})$ & 1/LR $(n=9)$ & $P$ value \\
\hline Age at time of operation (y) & $46.8 \pm 7.3(36-56)$ & $42.9 \pm 11.3(20-61)$ & .327 \\
\hline Gender, male & $7(70)$ & $9(100)$ & .211 \\
\hline Connective tissue disease & $1(10)$ & 0 & 1.000 \\
\hline \multicolumn{4}{|l|}{ Previous cardiac operation } \\
\hline Coarctation repair & 0 & $2(22)$ & .211 \\
\hline \multicolumn{4}{|l|}{ Preoperative dimensions $(\mathrm{cm})$} \\
\hline Annulus & $29.1 \pm 4.7(23-37)$ & $30.2 \pm 4.3(25-37)$ & .696 \\
\hline Sinuses & $48.3 \pm 9.4(35-65)$ & $46.5 \pm 9.2(28-55)$ & .825 \\
\hline Proximal ascending aorta & $47.8 \pm 5.4(39-57)$ & $46.7 \pm 8.7(35-58)$ & 1.000 \\
\hline Maximum & $53.1 \pm 5.2(47-65)$ & $51.8 \pm 5.9(38-58)$ & .513 \\
\hline \multicolumn{4}{|l|}{ Concomitant procedures } \\
\hline Aortic valve repair & $5(55)$ & $6(66)$ & .650 \\
\hline Arch replacement & $6(66)$ & $5(55)$ & 1.000 \\
\hline Other & 0 & $1(11)^{*}$ & \\
\hline \multicolumn{4}{|l|}{ Postrepair dimensions $(\mathrm{cm})$} \\
\hline Annulus & $21.8 \pm 1.2(20-24)$ & $24.6 \pm 1.2(20-31)$ & .115 \\
\hline Sinuses & $33.3 \pm 2.9(27-34)$ & $34.2 \pm 1.9(32-38)$ & .960 \\
\hline Ascending aorta & $24.2 \pm 2.0(20-26)$ & $24.7 \pm 1.7(22-28)$ & .826 \\
\hline Postrepair cusp angle & $165 \pm 10(134-180)$ & $133 \pm 16(105-177)$ & .001 \\
\hline \multicolumn{4}{|l|}{ Complications } \\
\hline Aortic dissection & $1(10)$ & 0 & 1.000 \\
\hline Age at time of study (y) & $50.3 \pm 8.3(40-64)$ & $46.9 \pm 11.6(22-62)$ & .838 \\
\hline Months after operation & $48.8 \pm 31.4(6-96)$ & $53.3 \pm 34.7(22-110)$ & .838 \\
\hline Aortic regurgitation on $\mathrm{TTE} \dagger$ & & & .221 \\
\hline 0 & $2(20)$ & $4(44)$ & \\
\hline 1 & $7(70)$ & $3(33)$ & \\
\hline 2 & 0 & $1(11)$ & \\
\hline \multicolumn{4}{|l|}{ Aortic stenosis on TTE } \\
\hline $\mathrm{V}_{\max }(\mathrm{m} / \mathrm{s})$ & $1.80 \pm 0.52(1.2-2.7)$ & $2.66 \pm 0.68(1.6-3.5)$ & .018 \\
\hline Mean PG (mm Hg) & $7.7 \pm 4.3(3-16)$ & $24.0 \pm 7.5(5-29)$ & .015 \\
\hline Maximum PG (mm Hg) & $14.3 \pm 9.0(5-28)$ & $29.9 \pm 13.3(10-48)$ & .017 \\
\hline
\end{tabular}

associated with decreased relative WSS along the outer curvature (Figure 2). In 12 patients, there was only 1 helix, a right-handed or counterclockwise helix (average grade $=1.8 \pm 0.7$ ). Among the remaining 5 patients ( 2 Sievers' type 0/LAT patients, and 3 type 1/LR patients), there were 2 countervailing helices, always with the right-handed helix being larger than the left-handed helix. Figure 3 demonstrates a Sievers' type 1/LR patient with a single right-handed helix in the ascending aorta, along with an associated nested helix forming during systole, and asymmetric increased WSS along the outer curvature.

At the time of imaging, 1 patient had a type B aortic dissection, with the dissection flap beginning immediately distal to the left subclavian artery. This patient had a Sievers' type 0/LAT valve with a large vortical flow pattern in the proximal descending aorta, which led into a dilated descending aorta with disorganized flow (Figure 4).

\section{Quantitative Flow and Shear-Stress Analysis}

The ratio of estimated WSS along the outer curvature to along the inner curvature was greater in the Sievers' type 1/LR patients compared with 0/LAT patients in the proximal and mid-ascending aorta (Table 2; Figure 5). Average and maximum WSS ratios were also greater in the Sievers' type 1/LR patients compared with 0/LAT patients. No statistically significant differences in jet eccentricity were found between Sievers' type 1/LR and 0/LAT patients in the ascending aorta. The cusp angle of 1/LR patients was significantly more acute than that for 0/LAT patients $(133 \pm 16$ vs $165 \pm 10$ degrees, $P=.001$; Table 1), but no statistically significant correlations were found between cusp angle and WSS, eccentricity, or peak gradient.

Relative to unoperated, control tricuspid patients, Sievers' type 1/LR patients had a greater WSS ratio in the mid-ascending aorta $(4.5 \pm 2.4$ vs $1.2 \pm 1.2, P=.007$ on post hoc testing). Conversely, the WSS ratio of Sievers' 
TABLE 2. Outcome measures

\begin{tabular}{lccc}
\hline \multicolumn{1}{c}{ Measure } & 0/LAT $(\mathbf{n}=\mathbf{1 0})$ & $\mathbf{1 / L R}(\mathbf{n}=\mathbf{9})$ & $\boldsymbol{P}$ value \\
\hline Helical flow & & & \\
$\quad$ Directionality & & & .271 \\
$\quad$ Right-handed & $8(80)$ & $4(44)$ & \\
$\quad$ Right- and left-handed & $2(20)$ & $3(33)$ & \\
\% lumen & $48 \pm 27$ & $45 \pm 38$ & .423 \\
$\quad$ Vortex grade & $0.60 \pm 0.97$ & $0.22 \pm 0.67$ & .326 \\
$\quad$ Helical rotation grade & $1.80 \pm 0.79$ & $1.44 \pm 1.01$ & .489 \\
Wall shear stress & & & \\
$\quad$ Proximal ascending & $2.1 \pm 0.9$ & $3.8 \pm 1.6$ & .009 \\
$\quad$ Mid-ascending & $2.4 \pm 1.3$ & $4.5 \pm 2.4$ & .027 \\
Distal ascending & $2.8 \pm 1.6$ & $4.9 \pm 3.5$ & .142 \\
Average & $2.4 \pm 0.7$ & $4.4 \pm 1.6$ & .004 \\
$\quad$ Maximum & $3.5 \pm 1.5$ & $6.3 \pm 3.1$ & .022 \\
Eccentricity & & & \\
$\quad$ Proximal ascending & $0.09 \pm 0.05$ & $0.12 \pm 0.05$ & .165 \\
$\quad$ Mid-ascending & $0.09 \pm 0.07$ & $0.11 \pm 0.07$ & .624 \\
Distal ascending & $0.13 \pm 0.10$ & $0.13 \pm 0.10$ & .462 \\
\hline
\end{tabular}

Values are $\mathrm{n}(\%)$, or mean $\pm \mathrm{SD}$ (range), unless otherwise indicated. O/LAT, Type 0 valve without the presence of a raphe, and with the 2 commissures oriented right-anterior-to-left-posterior; $1 / L R$, type 1 valve with fusion of the left and right cusps.

type 0/LAT patients was not significantly different from that of normal tricuspid patients.

In the Sievers' type 1/LR patients, 3 patients had mild-moderate aortic stenosis; therefore, the analyses were repeated excluding these patients. As before, the WSS ratio was greater in the Sievers' type 1/LR patients, compared with 0/LAT patients, in the proximal ascending aorta $(2.1 \pm 0.9$ for $0 / \mathrm{LAT}$ vs $3.7 \pm 2.0$ for $1 / \mathrm{LR})$, although this was now a trend, with $P=.065$, and the difference in the mid-ascending aorta was no longer statistically significant $(2.4 \pm 1.3$ vs $3.5 \pm 1.4, P=.159)$. The maximum WSS ratio tended to be higher in the Sievers' type 1/LR patients, compared with 0/LAT patients
$(3.5 \pm 1.5$ for $0 / \mathrm{LAT}$ vs $6.0 \pm 3.3$ for $1 / \mathrm{LR}, P=.065)$, and the average WSS ratio was statistically significantly greater in the Sievers' type 1/LR patients $(2.4 \pm 0.7$ for 0/LAT vs $4.0 \pm 1.3$ for $1 / \mathrm{LR}, P=.017$ ), consistent with the analysis of the complete cohort. Jet eccentricity was not significantly different between the 2 groups. Although the WSS in the mid-ascending aorta of the BAV and tricuspid groups were statistically significantly different on Kruskal-Wallis testing after removal of the aortic stenosis patients, post hoc testing comparing individual groups was no longer statistically significant secondary to diminished sample size.

\section{DISCUSSION}

This study is the first to examine systematically the role of BAV cusp morphology on ascending aortic flow after V-SARR. These observations add to our understanding of how BAV morphology relates to hemodynamics, as well as the potential role these abnormalities may play in the genesis of postoperative aortic complications. Patients with Sievers' type 1/LR valves after V-SARR demonstrated greater asymmetric WSS compared with patients with Sievers' type 0/LAT valves, and greater asymmetric WSS compared with control, unoperated tricuspid patients. Given the role of WSS in vascular remodeling, ${ }^{20}$ these flow perturbations may predispose patients to postoperative downstream thoracic aortic dilation or dissection.

These findings are consistent with prior reports of unoperated patients with Sievers' type 1/LR BAVs demonstrating abnormal flow and WSS. ${ }^{3-6,10}$ Barker and colleagues $^{5}$ studied 4D flow, in 12 Sievers' type 1/LR and 3 Sievers' type 1/RN patients, and found higher WSS in Sievers' type 1/LR patients both with and without stenosis, compared with healthy age- and aortic-matched controls. The maximal WSS was in the anterior and right anterior

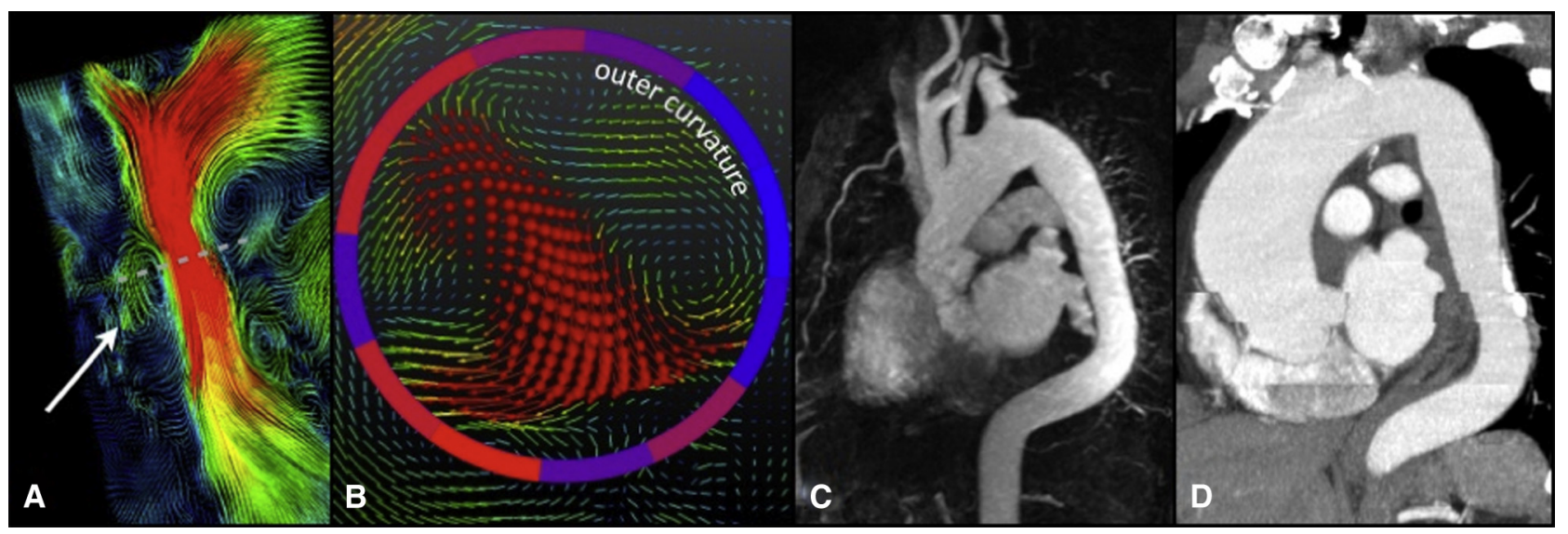

FIGURE 2. Example of flow in a patient with a Sievers' type 0/LAT BAV. Using streamlines, vortical flow can be seen (A, arrow) along the outer curvature of the aorta, above the level of the sinotubular junction. Vector fields demonstrate decreased WSS along the outer curvature (B). Although the WSS along the outer curvature is mildly decreased, the jet is still eccentrically placed along the inner curvature. Preoperative CT angiogram (D) and post-V-SARR maximum intensity MR angiogram (C) is provided for comparison. 


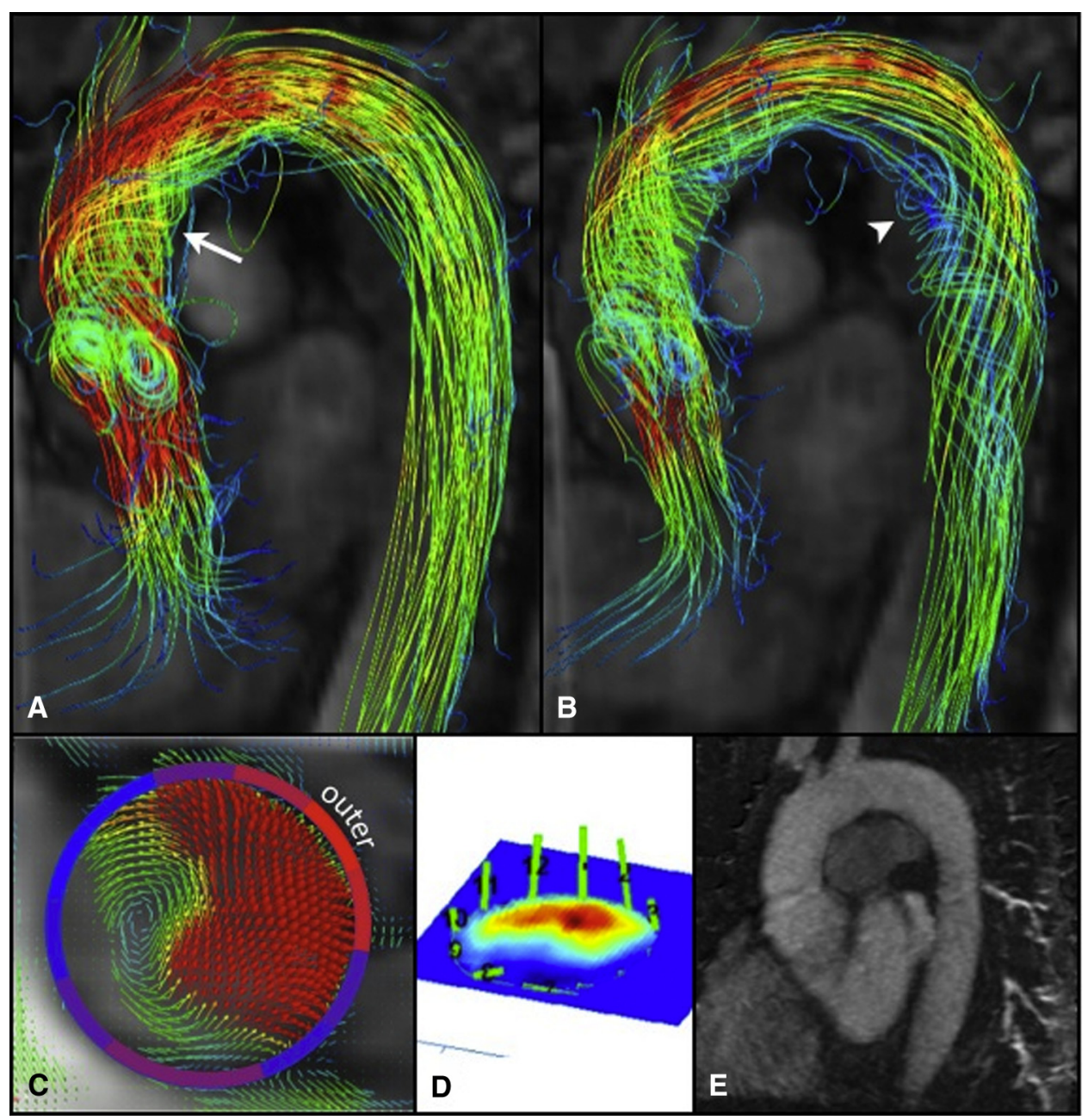

FIGURE 3. An example of a Sievers' type 1/LR BAV patient with eccentric flow in the ascending aorta. Streamlines demonstrate increased velocities along the outer curvature during peak systole (A) with a slower velocity nested helix along the inner curvature (A, arrow). Later during systole, the inner nested helix extends into the proximal descending aorta along the inner curvature (B, arrowhead). Vector fields in the mid-ascending aorta demonstrate an eccentrically placed jet with minimal retrograde flow along the inner curvature during peak systole (C), which translates to high WSS along the outer curvature (D). A maximum-intensity projection image from the postoperative MR angiogram is provided (E).

region, corresponding to the eccentric jets seen in those patients. ${ }^{5}$ Our finding of right-handed helical flow is also consistent with previous reports of 4D flow in Sievers' type $1 / \mathrm{LR}$ patients. ${ }^{3,6}$

Several analyses of 4D flow patterns in BAV patients have included Sievers' type 1/RN (fusion of the right and noncoronary cusps) valves. Bissell and colleagues ${ }^{6}$ analyzed 4D flow in 95 unoperated BAV patients (55 Sievers' type 1/LR, 33 Sievers' type 1/RN) who had varying degrees of aortic dilation and valve dysfunction, and 47 healthy volunteers. The BAV patients had greater WSS eccentricity compared with the healthy volunteers, with a correlation between aortic jet angle and WSS. Sievers' type 1/RN patients demonstrated flow angles, quantified rotational flow, and WSS that were greater than those in Sievers' type 1/LR patients ${ }^{6}$; however, the Sievers' type 1/RN BAV patients had more aortic dilation and more severe hemodynamic valvular abnormalities than did type 1/LR patients, which may have influenced their results.

A similar investigation of Sievers' type 1/LR and 1/RN $\mathrm{BAV}$ patients found elevated WSS in the right-anterior aorta in the type 1/LR patients and in the right-posterior aorta of type $1 / \mathrm{RN}$ patients, corresponding with the eccentric jets in the respective groups. ${ }^{21}$ In addition, they noted differences in aortopathy patterns between the groups: The majority $(87 \%)$ of Sievers' type 1/LR patients demonstrated dilation of the tubular ascending aorta and Sievers' type 1/RN patients demonstrated dilation of the aortic root $(53 \%)$ or diffuse dilation including the arch (34\%). ${ }^{21}$ In their study, displacement of the flow jet from the center of the aorta was the most sensitive parameter for distinguishing between types of aortopathy. ${ }^{21}$ Although the orientation of the aortic orifice relative to the ascending aorta in Sievers' type $1 / \mathrm{RN}$ patients is similar to $0 / \mathrm{LAT}, 1 / \mathrm{RN}$ 


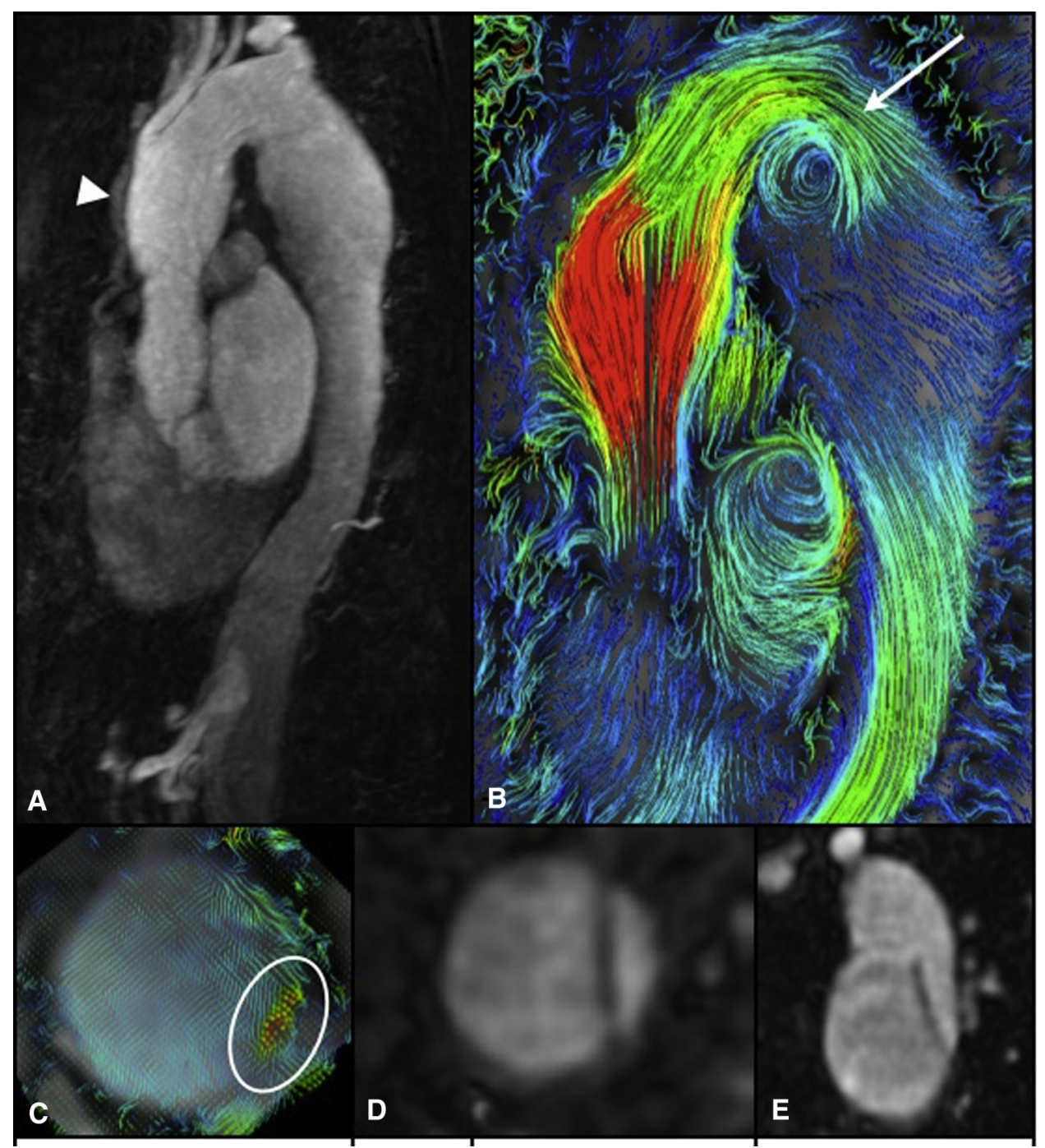

FIGURE 4. One Sievers' type 0/LAT BAV patient had a type B aortic dissection. A maximum intensity projection image from an MR angiogram (A) demonstrates dilation of the proximal descending thoracic aorta. Streamlines show high velocities in the ascending aorta distal to the graft, which leads into vortical flow within the false lumen in the proximal descending aorta (B, arrow). There is loss of signal (dark blue streamlines) in the dilated descending aorta, owing to turbulence. Vector fields in the descending aorta demonstrate increased velocities in the true lumen of the aorta (C, circle), and an axial plane through the descending aorta and transverse aorta (D and E) depict the origination of the dissection flap.

patients have a fused raphe and often different cusp angles that likely lead to distinct ascending aortic flow patterns relative to 0/LAT patients analyzed in the present study.

One other study has analyzed 4D flow in BAV patients after V-SARR; however, the small number of patients $(\mathrm{n}=7)$ precluded analysis of the effect of BAV morphology on flow patterns. Semaan and colleagues ${ }^{22}$ examined $4 \mathrm{D}$ flow in 7 BAV patients (1 Sievers' type 0/LAT, 1 Sievers' type 0 bicuspid aortic valve, 5 Sievers' type 1/LR), and 6 tricuspid patients who had undergone V-SARR, and compared them with age-matched and aortic-aneurysmmatched unoperated control patients. The BAV patients demonstrated eccentric flow preoperatively that decreased after V-SARR, but peak systolic velocities in the proximal aorta were higher. ${ }^{22}$ Flow velocities in tricuspid patients also increased after V-SARR, albeit to a lesser extent than those in the BAV patients. ${ }^{22}$ Comparisons with healthy, unoperated tricuspid subjects were not made.

In 0/LAT BAV patients, 4D flow patterns have not been systematically analyzed previously. In the present study, the finding of decreased WSS along the outer curvature in Sievers' type 0/LAT BAV, compared with Sievers' type $1 / \mathrm{LR}$, patients is likely due to the parallel orientation of the valve relative to the outer curvature; by contrast, in Sievers' type 1/LR patients, the fused cusps direct flow toward the outer curvature. Insertion and free-margin lengths of both cusps are nearly equal in Sievers' type 0 valves, leading to a balanced $180^{\circ} / 180^{\circ}$ configuration, 


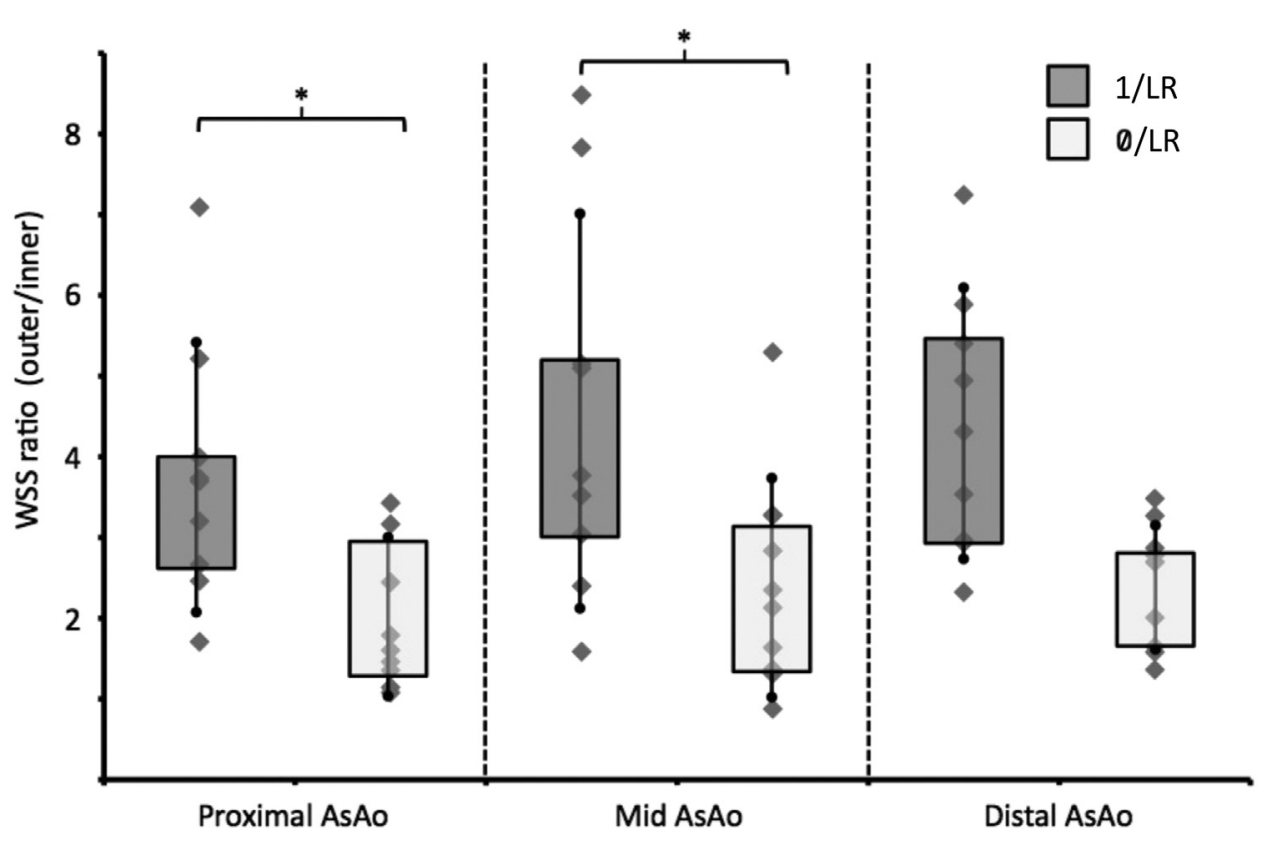

FIGURE 5. Ratio of the estimated WSS along the outer curvature over the inner curvature, at 3 levels along the AsAo (proximal, mid, and distal). Compared with Sievers' type 0/LAT patients, 1/LR patients had greater WSS ratios in the proximal and mid-ascending aorta $(* P<.05)$. Box plots represent upper and lower quartiles; whisker plots represent \pm 1 standard deviation and the gray diamonds represent individual WSS ratios. 1/LR, Type 1 valve with fusion of the left and right cusps; O/LAT, type 0 valve without the presence of a raphe, and with the 2 commissures oriented right-anterior-to-left-posterior; WSS, wall shear stress; AsAo, ascending aorta.

whereas Sievers' type 1 valves have a longer insertion length of the fused cusps and an unbalanced, asymmetric root geometry that can lead to decreased cusp mobility, often accentuated by a fibrotic raphe, resulting in an eccentric jet. Indeed, in the present study, the cusp angle of the 1/LR patients was significantly more acute than that in the 0/LAT patients (133 \pm 16 vs $165 \pm 10$ degrees). Thus, Sievers' types 1 and 0 BAVs are distinct entities anatomically and pathophysiologically, with different implications.

Indeed, we found flow patterns in Sievers' type 0/LAT patients that were not different from those in normal tricuspid patients. Although more longitudinal data are needed, this may affect the long-term risk of distal complications relative to Sievers' type $1 \mathrm{BAV}$ patients. Studies have suggested that Sievers' type 0 valves may demonstrate greater durability after V-SARR, compared with Sievers' type 1 valves, possibly related to improved flow dynamics. ${ }^{16}$

At Stanford, preserving bicuspid valves in young patients is considered only if the cusps are mobile and not excessively fibrotic or thickened. Although the Sievers' type 0/LAT patients in this study had lower WSS ratios relative to the type 1/LR BAV patients, all had ascending aortic aneurysms prompting surgical intervention. If WSS were the only reason for aortic dilation, one might expect these patients to present much later in life than those with Sievers' type 1/LR BAVs; however, in our study, there was no difference between groups in terms of age at time of operation.

One patient developed a type B aortic dissection postoperatively, before the 4D MRI flow study was performed; it is unknown whether the flow abnormalities seen in this study were present before the dissection, and predisposed the patient to dissection, or developed after the dissection. The patient did have a flow vortex in the proximal descending aorta, which has been described in V-SARR patients before dissection. ${ }^{15}$ This result is consistent with the findings of Francois and colleagues, ${ }^{23}$ who studied 4D flow in 13 patients with type B aortic dissections (including 3 patients with Marfan syndrome, and 1 patient with a BAV) who dissected after various open and endovascular aortic repairs (including 5 V-SARR). Eleven of 13 of these patients demonstrated additional vortices, including along the inner curvature of the proximal descending aorta. ${ }^{23}$ Although $4 \mathrm{D}$ flow studies have not demonstrated a direct relationship between altered flow pattern and aortic dissection, the degree of jet eccentricity ${ }^{11}$ and of restricted cusp motion ${ }^{24}$ have correlated with progression of aortic dilation in BAV patients, and altered aortic wall composition has been noted at the site of jet impact. ${ }^{25}$ Following the patients we imaged for another 5 to 10 years may elucidate which flow patterns predict distal aortic complications, because the native aorta distal to the graft is exposed to the perturbed WSS. Interestingly, WSS values increased from proximal to distal 
aorta, indicating that the eccentrically increased WSS is highest in the region of the native aortic arch.

\section{Limitations}

The implications of this investigation are limited by the small sample size and the selection bias for BAV patients who had had aortic root and ascending aortic aneurysms that required operation. Specifically, given the small sample size, the lack of detected differences between groups may be secondary to inadequate power, and any conclusions regarding equivalency should be made with caution. The preserved BAVs were functioning well without severe stenosis or regurgitation. Without 4D flow measurements before V-SARR, we do not know whether these flow patterns were present preoperatively and how they related to their preoperative aortic dilation. Flow may have been affected by cusp repair, although that would be expected to predominantly affect diastolic flow patterns.

Additionally, calculated WSS values are prone to error, and therefore, relative values were used in this analysis. Furthermore, these WSS values described the forces on the intimal surfaces, and strains imparted on the media cannot be quantified using 4D flow. Lastly, although the very small number of postoperative aortic complications over 5 to 10 years among BAV patients after V-SARR is reassuring, ${ }^{12}$ longer follow-up is required to determine how these blood flow and WSS abnormalities relate to downstream aortic complications.

In conclusion, this analysis demonstrates that BAV cusp morphology significantly affects flow patterns and WSS in patients after V-SARR. Although Sievers' type 1/LR BAV patients had higher WSS and more flow eccentricity than control patients, the WSS for Sievers' type 0/LAT BAV patients was not different from control tricuspid patients. These results suggest that Sievers' type 1/LR $\mathrm{BAV}$ patients may be at higher risk for long-term complications, although clearly longer follow-up is required.

\section{Conflict of Interest Statement}

D. Craig Miller is a consultant for Abbott Vascular, Medtronic, and GENTAC (NHLBI), and is on the Executive Committee for the PARTNER Trial (Edwards LifeSciences, LLP, nonremunerative). The other authors have nothing to disclose with regard to commercial support.

The authors thank Andrew Wentland for helping with alias correction, and Anne Sawyer, Kevin Epperson, and Hadas Shiran, MD, for their assistance in performing the 4D flow studies. The authors appreciate the help of Zoe Magee, RN, for assistance in obtaining patient follow-up, and Dr Christine Mauro, PhD, Department of Statistics, Columbia University, for her statistical expertise.

\section{References}

1. Fazel SS, Mallidi HR, Lee RS, Sheehan MP, Liang D, Fleischman D, et al. The aortopathy of bicuspid aortic valve disease has distinctive patterns and usually involves the transverse aortic arch. J Thorac Cardiovasc Surg. 2008; 135:901-7.

2. Hahn RT, Roman MJ, Mogtader AH, Devereux RB. Association of aortic dilation with regurgitant, stenotic and functionally normal bicuspid aortic valves. J Am Coll Cardiol. 1992;19:283-8.

3. Hope MD, Hope TA, Meadows AK, Ordovas KG, Urbania TH, Alley MT, et al Bicuspid aortic valve: four-dimensional MR evaluation of ascending aortic systolic flow patterns. Radiology. 2010;255:53-61.

4. Barker AJ, Lanning C, Shandas R. Quantification of hemodynamic wall shear stress in patients with bicuspid aortic valve using phase-contrast MRI. Ann Biomed Eng. 2010;38:788-800.

5. Barker AJ, Markl M, Burk J, Lorenz R, Bock J, Bauer S, et al. Bicuspid aortic valve is associated with altered wall shear stress in the ascending aorta. Circ Cardiovasc Imaging. 2012;5:457-66.

6. Bissell MM, Hess AT, Biasiolli L, Glaze SJ, Loudon M, Pitcher A, et al. Aortic dilation in bicuspid aortic valve disease: Flow pattern is a major contributor and differs with valve fusion type. Circ Cardiovasc Imaging. 2013; 6:499-507.

7. Kang JW, Song HG, Yang DH, Baek S, Kim DH, Song JM, et al. Association between bicuspid aortic valve phenotype and patterns of valvular dysfunction and bicuspid aortopathy: comprehensive evaluation using MDCT and echocardiography. JACC Cardiovasc Imaging. 2013;6:150-61.

8. Della Corte A, Bancone C, Buonocore M, Dialetto G, Covino FE, Manduca S, et al. Pattern of ascending aortic dimensions predicts the growth rate of the aorta in patients with bicuspid aortic valve. JACC Cardiovasc Imaging. 2013;6: 1301-10.

9. Russo CF, Cannata A, Lanfranconi M, Vitali E, Garatti A, Bonacina E. Is aortic wall degeneration related to bicuspid aortic valve anatomy in patients with valvular disease? J Thorac Cardiovasc Surg. 2008;136:937-42.

10. Hope MD, Hope TA, Crook SE, Ordovas KG, Urbania TH, Alley MT, et al 4d flow CMR in assessment of valve-related ascending aortic disease. JACC Cardiovasc Imaging. 2011;4:781-7.

11. Hope MD, Wrenn J, Sigovan M, Foster E, Tseng EE, Saloner D. Imaging biomarkers of aortic disease: increased growth rates with eccentric systolic flow. J Am Coll Cardiol. 2012;60:356-7.

12. Kari FA, Liang DH, Kvitting JP, Stephens EH, Mitchell RS, Fischbein MP, et al Tirone David valve-sparing aortic root replacement and cusp repair for bicuspid aortic valve disease. J Thorac Cardiovasc Surg. 2013;145:S35-40.

13. Demers P, Miller DC. Simple modification of "T. David-V" valve-sparing aortic root replacement to create graft pseudosinuses. Ann Thorac Surg. 2004;78: 1479-81.

14. Sievers HH, Schmidtke C. A classification system for the bicuspid aortic valve from 304 surgical specimens. J Thorac Cardiovasc Surg. 2007;133:1226-33.

15. Hope TA, Kvitting JP, Hope MD, Miller DC, Markl M, Herfkens RJ. Evaluation of marfan patients status post valve-sparing aortic root replacement with $4 \mathrm{~d}$ flow. Magn Reson Imaging. 2013;31:1479-84.

16. Kari FA, Kvitting JP, Stephens EH, Liang DH, Merk DR, Fischbein MP, et al Tirone David procedure for bicuspid aortic valve disease: impact of root geometry and valve type on mid-term outcomes. Interact Cardiovasc Thorac Surg. 2014;19:375-81

17. Hsiao A, Lustig M, Alley MT, Murphy M, Chan FP, Herfkens RJ, et al. Rapid pediatric cardiac assessment of flow and ventricular volume with compressed sensing parallel imaging volumetric cine phase-contrast MRI. AJR Am J Roentgenol. 2012;198:W250-259.

18. Geiger J, Arnold R, Herzer L, Hirtler D, Stankovic Z, Russe M, et al. Aortic wall shear stress in marfan syndrome. Magn Reson Med. 2013;70:1137-44.

19. Stalder AF, Russe MF, Frydrychowicz A, Bock J, Hennig J, Markl M. Quantitative 2d and 3d phase contrast MRI: optimized analysis of blood flow and vessel wall parameters. Magn Reson Med. 2008;60:1218-31.

20. Lehoux S, Tedgui A. Cellular mechanics and gene expression in blood vessels. J Biomech. 2003;36:631-43.

21. Mahadevia R, Barker AJ, Schnell S, Entezari P, Kansal P, Fedak PW, et al Bicuspid aortic cusp fusion morphology alters aortic $3 \mathrm{~d}$ outflow patterns, wall shear stress and expression of aortopathy. Circulation. 2014;129:673-82.

22. Semaan E, Markl M, Malaisrie SC, Barker A, Allen B, McCarthy P, et al Haemodynamic outcome at four-dimensional flow magnetic resonance imaging following valve-sparing aortic root replacement with tricuspid and bicuspid valve morphology. Eur J Cardiothorac Surg. 2014;45:818-25. 
23. Francois CJ, Markl M, Schiebler ML, Niespodzany E, Landgraf BR, Schlensak C, et al. Four-dimensional, flow-sensitive magnetic resonance imaging of blood flow patterns in thoracic aortic dissections. J Thorac Cardiovasc Surg. 2013;145:1359-66

24. Della Corte A, Bancone C, Conti CA, Votta E, Redaelli A, Del Viscovo L, et al. Restricted cusp motion in right-left type of bicuspid aortic valves: a new risk marker for aortopathy. J Thorac Cardiovasc Surg. 2012;144:360-9.
25. Girdauskas E, Rouman M, Disha K, Scholle T, Fey B, Theis B, et al. Correlation between systolic transvalvular flow and proximal aortic wall changes in bicuspid aortic valve stenosis. Eur J Cardiothorac Surg. 2014;46:234-9.

Key Words: Bicuspid aortic valve, valve-sparing aortic root replacement, 4D flow MRI, thoracic aorta

\title{
EDITORIAL COMMENTARY
}

\section{How pathologic is the function of a bicuspid aortic valve?}

\author{
Hans-Joachim Schäfers, MD
}

See related article on pages 59-68.

In the past 20 years, the attitude of surgeons to the bicuspid aortic valve (BAV) has changed dramatically. Initially, this valve form was considered to be pathologic in form and function by nature, ${ }^{1}$ justifying its liberal replacement. With time the BAV has gained increasing interest, in part because of its associated aortopathy. ${ }^{2}$ It has also been recognized that the prognosis is not uniformly poor, both in its spontaneous course ${ }^{3}$ and after repair, ${ }^{4}$ and that BAV anatomy is not uniform but varies with respect to degree of fusion, fusion pattern, and also commissural orientation. ${ }^{5}$ The analysis of repair failures has pointed out the clinical relevance of the anatomic variability of $\mathrm{BAV}{ }^{4}$ The aortopathy seen with BAV has been the subject of controversial discussion with regard to whether it is the result of abnormal turbulence characteristic for the BAV or related to genetic abnormalities of the aortic wall that are related to BAV morphology, such as alterations in the endothelial nitric oxide synthase pathway. ${ }^{6}$

The study of Stephens and colleagues ${ }^{7}$ in this issue of the Journal contributes additional information on the abnormal flow pattern in the ascending aorta, thus seemingly reinforcing the possible relationship between

From the Department of Thoracic and Cardiovascular Surgery, University Hospitals, Homburg/Saar, Germany.

Disclosures: Author has nothing to disclose with regard to commercial support.

Received for publication April 19, 2015; accepted for publication April 24, 2015.

Address for reprints: Hans-Joachim Schäfers, MD, Department of Thoracic and Car-

diovascular Surgery, University Hospitals, Kirrberger Strasse, 66421 Homburg/

Saar, Germany (E-mail: j.schaefers@uniklinikum-saarland.de or h-j.schaefers@

uks.eu).

J Thorac Cardiovasc Surg 2015; 150:68-9

$0022-5223 / \$ 36.00$

Copyright (c) 2015 by The American Association for Thoracic Surgery

http://dx.doi.org/10.1016/j.jtcvs.2015.04.043 aneurysm formation and turbulence. More importantly, the study shows that the presence of turbulence and its severity is related to the morphology of the valve. Patients who had undergone valvesparing aortic replacement were analyzed according to their type of BAV (ie, no raphe, considered type 0 , or asymmetric with 1

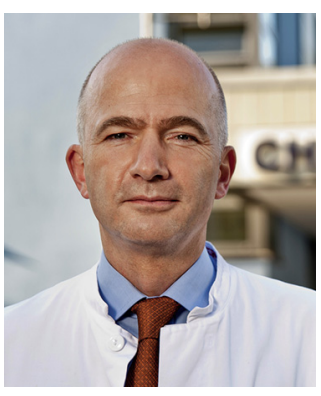
raphe). ${ }^{8}$ Stephens and colleagues ${ }^{7}$

found the expected turbulence, with a maximum toward the outer curvature of the tubular ascending aorta with type 1 valves, whereas in patients with type 0 BAV more laminar flow patterns were observed. They concluded that the increased turbulence may be related to a higher probability of secondary aortic complications after valvepreserving surgery in type $1 \mathrm{BAV}$.

Stephens and colleagues ${ }^{7}$ have generated interesting and important physiologic data regarding flow in the ascending aorta and its possible relationship with aneurysm formation. An even more fascinating dimension of the study is the documentation that symmetric configuration of a BAV leads to flow patterns across the valve and in the ascending aorta that are close to normal. Thus the findings provide new evidence that the anatomic configuration of the BAV is related to its physiologic function. This implies that in reconstruction or preservation of the BAV a symmetric configuration of the commissures should be ideal, as has been concluded previously on the basis of repair durability. ${ }^{4}$

There are limitations of the study that have to be kept in mind, however, and questions remain unanswered. Does the study prove a causal relationship between turbulence and aneurysm formation? Will less turbulence with symmetric orientation of commissures actually result in less aneurysm formation? How high is the probability of downstream 